\title{
Métodos não farmacológicos no alívio da dor relacionada ao rastreamento e diagnóstico do câncer de mama: revisão de escopo
}

Non-pharmacological methods in the relief of pain related to the screening and diagnosis of breast cancer: scope review

Métodos no farmacológicos para el alivio del dolor relacionado con el examen y el diagnóstico del cáncer de mama: revisión del alcance

\section{Resumo}

Objetivo: mapear na literatura as terapias não farmacológicas utilizadas no alívio da dor em mulheres submetidas ao rastreamento e diagnóstico do câncer de mama. Metodologia: estudo do tipo revisão de escopo realizado em nove fontes de dados através dos descritores mamografia, biopsia por agulha, terapias complementares e dor. Dois pesquisadores independentes realizaram a busca dos estudos e um pesquisador aplicou os critérios de inclusão e exclusão e realizou a análise. Foram incluídos os estudos que respondiam à questão de pesquisa, totalizando doze publicações. A extração dos dados da pesquisa foi executada através de instrumentos construídos pelos autores que permitiram a categorização das informações apresentadas. Resultados: dentre a amostra final de doze estudos, os métodos não farmacológicos utilizados foram musicoterapia, meditação, hipnose, exercícios físicos, intervenções de enfermagem e toque terapêutico. Observou-se, que a musicoterapia, os exercícios físicos e as intervenções de enfermagem foram utilizados na redução da dor durante a mamografia, e os demais métodos encontrados foram testados no procedimento de biópsia. Conclusão: evidenciou-se que os métodos não farmacológicos utilizados no alívio da dor durante o rastreamento e diagnóstico do câncer de mama são efetivos e podem contribuir para mudanças 
nos protocolos de atendimentos por serem uma ferramenta de fácil acesso, baixo custo, criativa, segura e que fornece benefícios às mulheres.

Palavras-chave: Mamografia; Biopsia por agulha; Dor; Manejo da dor; Analgesia; Terapias complementares.

\begin{abstract}
Objective: to map non-pharmacological therapies used in the literature to relieve pain in women undergoing breast cancer screening and diagnosis. Methodology: Scope review study carried out in nine data sources using the descriptors mammography, needle biopsy, complementary therapies and pain. Two independent researchers performed the search for the studies and one researcher applied the inclusion and exclusion criteria and performed the analysis. Studies that answered the research question were included, totaling twelve publications. The extraction of research data was performed using instruments built by the authors that allowed the categorization of the information presented. Results: among the final sample of twelve studies, the non-pharmacological methods used were music therapy, meditation, hypnosis, physical exercise, nursing interventions and therapeutic touch. It was observed that music therapy, physical exercises and nursing interventions were used to reduce pain during mammography, and the other methods found were tested in the biopsy procedure. Conclusion: it was shown that non-pharmacological methods used to relieve pain during screening and diagnosis of breast cancer are effective and can contribute to changes in care protocols as they are an easy-access, low-cost, creative, safe and that provides benefits to women.
\end{abstract}

Keywords: Mammography; Biopsy needle; Pain; Pain management; Analgesia; Complementary therapies.

\title{
Resumen
}

Objetivo: mapear las terapias no farmacológicas utilizadas en la literatura para aliviar el dolor en mujeres sometidas a cribado y diagnóstico de cáncer de mama. Metodología: Estudio de revisión de alcance realizado en nueve fuentes de datos utilizando los descriptores mamografía, biopsia con aguja, terapias complementarias y dolor. Dos investigadores independientes realizaron la búsqueda de los estudios y un investigador aplicó los criterios de inclusión y exclusión y realizó el análisis. Se incluyeron los estudios que respondieron a la pregunta de investigación, totalizando doce publicaciones. La extracción de los datos de la investigación se realizó mediante instrumentos construidos por los autores que permitieron la categorización de la información presentada. Resultados: entre la muestra final de doce estudios, los métodos no farmacológicos utilizados fueron musicoterapia, meditación, hipnosis, ejercicio físico, intervenciones de enfermería y toque terapéutico. Se observó que la musicoterapia, los ejercicios físicos y las intervenciones de enfermería se utilizaron para reducir el dolor durante la mamografía, y los demás métodos encontrados se probaron en el procedimiento de biopsia. Conclusión: se demostró que los métodos no farmacológicos utilizados para aliviar el dolor durante el cribado y diagnóstico de cáncer de mama son efectivos y pueden contribuir a cambios en los protocolos de atención por ser un método de fácil acceso, bajo costo, creativo, seguro y que brinda beneficios a mujeres.

Palabras clave: Mamografía; Biopsia con aguja; Dolor; Manejo del dolor; Analgesia; Terapias complementarias.

\section{Introdução}

O câncer de mama é considerado o segundo tipo de neoplasia de elevada taxa de mortalidade entre as mulheres. A mamografia é considerada o método diagnóstico de maior precisão através da detecção precoce da doença. Esse fator reflete na melhora do prognóstico e consequentemente maiores chances de cura. A exposição radiológica somada a compressão do tecido mamário permite a identificação de lesões impalpáveis e até mesmo o desenvolvimento do carcinoma in situ (Inca, 2019).

A compressão do tecido mamário durante a realização do exame mamográfico gera dor, o que pode contribuir para a não adesão das mulheres a essa ferramenta insubstituível (Almeida, Santana, Silva \& Melo, 2017; Fayanju, Kraenzle, Drake, Oka \& Goodman, 2014). Alguns fatores podem contribuir para uma maior sensibilidade dolorosa durante o procedimento de mamografia como as fases do ciclo menstrual, a característica anatômica e o volume mamário (Kitaoka \& Kawashima, 2018). Estudo realizado no intuito de diminuir essa sensibilidade dolorosa obteve resultado apenas em casos de relato de dor leve, através de agentes farmacológicos, como o paracetamol (Freitas-junior et al., 2018).

Somada aos achados do exame mamográfico, as biópsias mamárias, responsáveis pelo diagnóstico definitivo do câncer de mama, são classificadas de acordo com a técnica de realização, sendo elas biópsia com agulha central, aspiração por agulha fina e biópsia assistida a vácuo guiadas por exames de imagem como ultrassonografia, mamografia ou ressonância magnética (Gioia, Lima \& Maiato, 2017). 
A punção citológica aspirativa por agulha fina (PAAF) de massas mamárias, tem sido substituída por biópsia com agulha grossa guiada por ultrassom em muitos países. Essa prática não deve ser realizada uma vez que os resultados obtidos pela realização da citologia aspirativa por agulha fina apresentam detalhes citopatológicos importantes com menores custos (Abdel-Hadi, Abdel-Hamid, Abdel-Razek \& Fawzy, 2010).

Os requisitos para realização de uma PAAF ideal da mama incluem a fixação da lesão e agilidade no procedimento. A agulha deve ser movimentada rapidamente para trás e para frente através da punção, porém pode ser considerado a necessidade de novas perfurações para avaliação da lesão por outros ângulos. Vale considerar que para aspiração é utilizada agulha de calibre 22, 23, 25 ou 27, e a realização não exige o uso rotineiro de anestésicos locais na pele (Field, Raymond, Rickard \& Schmitt, 2020). Em relação a dor gerada pelo procedimento de citologia aspirativa por agulha fina, estudo identificou que o único fator significativo nos escores de dor foi o tamanho da agulha utilizada, a de calibre 21 (Satchithananda et al., 2005).

Diferentemente da PAAF, a biópsia por agulha grossa é realizada com um bisturi e agulha central através de um ponto de incisão guiada por exames de imagem como ultrassom, mamografia ou ressonância magnética. O procedimento é feito acompanhado de um anestésico local na pele (Field et al., 2020).

Estudo relatou aumento da dor relacionada às biópsias de mama guiadas por ressonância magnética (Pang, Crystal, Kulkarni, Murphy \& Menezes, 2016). Segundo Zagouri et al., (2008), biópsia de lesões impalpáveis são mais dolorosas do que as de lesões palpáveis. Estratégias de enfrentamento da dor devem ser consideradas diante da realização desses procedimentos diagnósticos além da conversa da equipe sobre a dor com as pacientes antes da biópsia. Espera-se que essas ações possam reduzir a ansiedade e a dor (Soo et al., 2014).

Terapias complementares, associadas a medicina tradicional, são capazes de reduzir a dor poupando até mesmo os altos custos dispensados aos tratamentos tradicionais. Algumas estratégias são utilizadas, como métodos baseados no corpo, terapias energéticas, terapia eletromagnética, preparações dietéticas e a base de ervas, terapias sensoriais, terapias de movimento e terapias mentais (Hamlin \& Robertson, 2017).

Terapias não farmacológicas sensoriais como a aromaterapia e a música podem diminuir a ansiedade e a dor sem efeitos secundários que possam interromper ou atrapalhar tratamentos primários, como foi evidenciado em estudo com pacientes com câncer de mama no perioperatório (Yangfan, Li, Xie, Xu \& Liu, 2018).

As terapias complementares, apresentam eficácia no alívio da dor e ansiedade em diversos cenários de saúde e por possuírem baixo custo para a sua implementação, atualmente estão dentro das práticas integrativas e complementares oferecidas ao indivíduo, família e coletividade como medida terapêutica no âmbito do Sistema Único de Saúde (SUS).

Diante do exposto, o objetivo desse estudo é mapear na literatura as terapias não farmacológicas utilizadas no alívio da dor em mulheres submetidas ao rastreamento e diagnóstico do câncer de mama. Acredita-se que essas terapias possam contribuir para melhor adesão das mulheres ao rastreamento e diagnóstico do câncer de mama pela diminuição da resposta à dor.

\section{Metodologia}

Trata-se de uma scoping review, elaborada através de métodos que permitem mapear as evidências disponíveis no intuito de determinar a amplitude de um determinado tema e até mesmo sugerir novas pesquisas (Tricco et al., 2018).

Para realização da pesquisa foram respeitados alguns passos necessários como: estabelecimento da questão da pesquisa; identificação de estudos relevantes; inclusão dos estudos selecionados; categorização dos dados obtidos; e agrupamento, síntese e descrição dos resultados (Peters et al., 2020).

Inicialmente foi executada uma busca ampliada na bibliografia científica, com o objetivo de identificar a existência de revisões com escopo semelhante aos critérios adotados por esse estudo, a fim de reduzir viés e evitar duplicadas. Para tal, 
foram consultadas as seguintes plataformas de registros internacionais de estudos científicos: International Prospective Register of Systematic Reviews (PROSPERO), Open Science Framework (OSF), The Cochrane Library, JBI Clinical Online Network of Evidence for Care and Therapeutics (COnNECT+) e Database of Abstracts of Reviews of Effects (DARE). Os resultados dessa busca evidenciaram a existência de um registro de revisão de escopo na Open Science Framework (OSF) com objetivo de realizar pesquisa apenas no rastreamento, o que justifica a importância deste estudo com métodos não farmacológicos no rastreamento e também no diagnóstico do câncer de mama.

A fim de definir a questão da pesquisa e os principais elementos da busca de dados desta revisão, utilizou-se a ferramenta metodológica do Joanna Briggs Institute (Peters et al., 2020) PCC (População, Conceito e Contexto), indicada para revisões de escopo. Foi denominado para população, mulheres submetidas à mamografia e biopsias mamárias; o conceito escolhido foi uso de métodos não farmacológicos e o contexto investigado foi a dor (Quadro 1). Para tanto, obteve-se o seguinte questionamento: quais os métodos não farmacológicos utilizados no alívio da dor em mulheres submetidas ao rastreamento e diagnóstico do câncer de mama?

Quadro 1 - Descritores e palavras-chaves utilizadas na busca. Aracaju, SE, Brasil, 2021.

\begin{tabular}{|c|c|c|}
\hline PCC & MESH/DeCS & Palavras-chave \\
\hline Population & Mammography/Mamografia & $\begin{array}{l}\text { Biopsy, } \\
\text { needle/Biopsia por } \\
\text { agulha }\end{array}$ \\
\hline \multicolumn{3}{|c|}{$A N D$} \\
\hline \multicolumn{3}{|c|}{$\begin{array}{c}\text { Complementary therapies/Terapias Complementares OR } \\
\text { Alternative medicine/Medicina alternativa OR Music } \\
\text { therapy/Musicoterapia OR Massage/Massagem OR } \\
\text { hypnosis OR Meditation/Meditação OR Breathing } \\
\text { exercises/Exercício de respiração OR } \\
\text { Acupuncture/Acupuntuta OR Yoga/Yoga OR } \\
\text { Homeopathy/Homeopatia OR Transcutaneous eletric } \\
\text { nerve stimulation OR Musculoskeletal } \\
\text { Manipulations/Manipulações Musculoesqueléticas } \\
\text { OR Aromatheraphy/Aromaterapia }\end{array}$} \\
\hline \multicolumn{3}{|c|}{$A N D$} \\
\hline Context & $\begin{array}{c}\text { Pain/Dor } \\
\text { OR } \\
\text { Pain management/Manejo da dor }\end{array}$ & Analgesia \\
\hline
\end{tabular}

Fonte: Autores (2021).

A busca dos estudos relevantes deu-se através das seguintes fontes de dados: Cummulative Index to Nursing and Allied Health Literature (CINAHL), Embase, Science direct, Bireme, Scopus, Web of Science, Medical Literature Analysis and Retrieval System Online (MEDLINE), Catálogo de Teses e Dissertações (CAPES) e Google Scholar.

Para busca dos estudos, utilizou-se os operadores booleano AND e OR de maneira restritiva e aditiva respectivamente, com os seguintes descritores indexados no MESH "complementary therapies", "alternative medicine", "aromatherapy", “yoga", "homeopathy", "Musculoskeletal Manipulations", "acupuncture”, "Meditation”, "Massage", "hypnosis ", "Breathing exercises", “music therapy", "pain", "pain management", “analgesia”, "mammography”, "biopsy needle". Foi construída uma estratégia de busca para cada base de dados escolhida utilizando esses descritores, conforme apresentada no Quadro 2. 
Quadro 2 - Sintaxe de busca nas fontes de dados. Aracaju, SE, Brasil, 2021.

\begin{tabular}{|c|c|}
\hline Fontes de dados & Sintaxe \\
\hline $\begin{array}{l}+P U B M E D / \\
\text { MEDLINE }\end{array}$ & 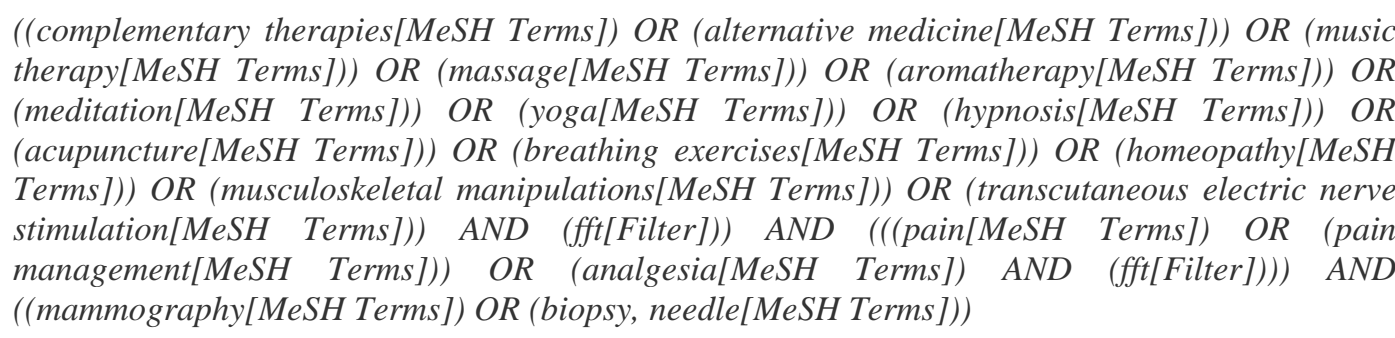 \\
\hline IL & $\begin{array}{l}\text { ( complementary therapies* OR alternative medicine* OR music therapy* OR massage OR } \\
\text { acupuncture OR meditation OR hypnosis OR breathing exercises* OR yoga OR aromatherapy OR } \\
\text { Transcutaneous electric nerve Stimulation* OR Musculoskeletal Manipulations* OR homeopathy) } \\
\text { AND ( pain OR pain management* OR analgesia) AND (mammography OR biopsy, needle*) }\end{array}$ \\
\hline Science direct & $\begin{array}{l}\text { ("alternative medicine" OR "music therapy" OR massage OR hypnosis OR meditation OR } \\
\text { "breathing exercises") AND "pain management" AND (mammography OR "biopsy, needle") }\end{array}$ \\
\hline BIREME & $\begin{array}{l}\text { ("complementary therapies" OR "music therapy" OR massage OR aromatherapy OR "alternative } \\
\text { medicine" OR hypnosis OR meditation OR "breathing exercises" OR acupuncture OR yoga OR } \\
\text { homeopathy OR "transcutaneous eletric nerve stimulation" OR "Musculoskeletal Manipulations") } \\
\text { AND (pain OR pain management OR analgesia) AND (mammography OR "biopsy, needle") }\end{array}$ \\
\hline EMBASE & 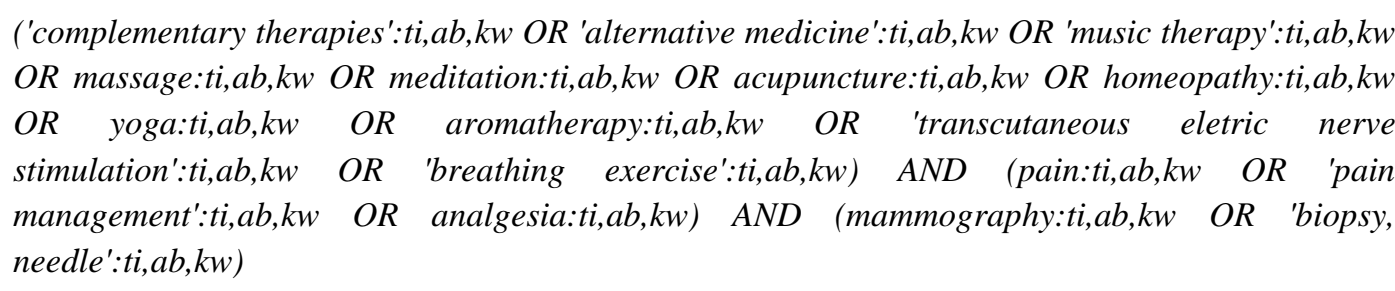 \\
\hline SCOPUS & $\begin{array}{l}\text { ( TITLE-ABS-KEY ( "complementary therapies" OR "alternative medicine" OR yoga OR } \\
\text { "Musculoskeletal Manipulations" OR "music therapy" OR aromatherapy OR massage OR } \\
\text { meditation OR hypnosis OR acupuncture OR "transcutaneous eletric nerve stimulation" OR } \\
\text { "breathing exercises") AND TITLE-ABS-KEY ( pain OR "pain management" OR analgesia) } \\
\text { AND TITLE-ABS-KEY ( mammography OR "biopsy, needle")) }\end{array}$ \\
\hline Web of Science & $\begin{array}{l}\text { TS= (("complementary therapies" OR "music therapy" OR massage OR aromatherapy OR } \\
\text { "alternative medicine" OR hypnosis OR meditation OR "breathing exercises" OR acupuncture OR } \\
\text { yoga OR homeopathy OR "transcutaneous eletric nerve stimulation" OR "Musculoskeletal } \\
\text { Manipulations") AND (pain OR "pain management" OR analgesia) AND (mammography* OR } \\
\text { "biopsy needle")) }\end{array}$ \\
\hline $\begin{array}{l}\text { Catálogo de Teses e } \\
\text { Dissertações } \\
\text { (CAPES) }\end{array}$ & $\begin{array}{l}\text { (mammography) AND pain AND ("complementary therapy" OR "alternative medicine" OR } \\
\text { "Physical Activity" OR music therapy OR aromatherapy OR meditation OR yoga OR hypnosis OR } \\
\text { acupuncture) }\end{array}$ \\
\hline Google Scholar & $\begin{array}{l}\text { (("biopsy, needle" OR mammography) AND "pain management" AND ("complementary therapies" } \\
\text { OR "music therapy" OR meditation OR massage OR hypnosis OR acupuncture OR "breathing } \\
\text { exercises" OR "transcutaneous eletric nerve stimulation")) }\end{array}$ \\
\hline
\end{tabular}


A busca dos registros nas bases de dados foi efetuada por meio do Portal de Periódicos da Coordenação de Aperfeiçoamento de Pessoal de Nível Superior (CAPES) através do acesso remoto ao conteúdo da Comunidade Acadêmica Federada (CAFe), recurso financiado pela Universidade Federal de Sergipe (UFS).

A escolha dos estudos deu-se através da aplicação dos seguintes critérios de inclusão: manuscritos disponíveis online na íntegra, dissertações, teses, Portarias ministeriais, guidelines e artigos científicos, sem recorte temporal, publicados em qualquer idioma, independente do método utilizado. Foram excluídos os estudos com texto completo indisponível e os que não responderam à questão de pesquisa. Os artigos duplicados nas fontes foram computados apenas uma vez.

A extração e categorização dos dados obtidos foram realizadas, simultaneamente, por dois autores, às cegas e de forma independente, utilizando-se dispositivos eletrônicos distintos, no mês de julho de 2021. Um terceiro autor foi consultado para decisão final nos casos de divergências ou dúvidas na seleção da amostra. Além disso, o terceiro autor também realizou uma busca manual a partir das referências dos registros incluídos.

Após a seleção dos estudos, foi utilizado um instrumento estruturado para síntese e descrição dos resultados com os seguintes itens: identificação do artigo, título, país/ano, fonte de dados, amostra do estudo, nível de evidência/grau de recomendação, tipo de método não farmacológico e desfecho.

No que se refere à classificação dos estudos quanto ao nível de evidência e grau de recomendação, foi utilizada a categorização protocolada pelo Oxford Centre for Evidence-based Medicine, que estabelece que quanto menor for o número determinado para um estudo, maior será o seu nível de evidência. Acresce a isso, o grau de recomendação "A" é conceituado maior e o "D" o de menor recomendação.

Com o propósito de garantir a integridade desse estudo e rigor metodológico, utilizou-se o checklist Preferred Reporting Items for Systematic reviews and Meta-Analyses extension for Scoping Reviews (PRISMA-ScR) (Tricco et al., 2018), em consonância com as recomendações fornecidas pelo Instituto Joanna Briggs (Peters et al., 2020), e foi registrada na plataforma OSF (https://osf.io/7gu8t), DOI 10.17605/OSF.IO/RAZHT.

\section{Resultados}

A pesquisa inicial obteve 1.191 estudos, desses: 940 no Google Scholar; 6 no Web of Science; 137 no Science Direct; 19 no MEDLINE, 13 no CINAHL; 27 na BIREME, 3 na EMBASE, 41 na SCOPUS e 5 no catálogo de dissertações e teses da CAPES. Após aplicação dos critérios de exclusão e inclusão, para primeira análise, foram avaliados 1.125 estudos a partir da leitura do título e resumo pelos pesquisadores.

Essa primeira leitura de títulos selecionou 44 estudos, sendo 20 computados apenas uma vez, por duplicidade nas fontes de dados. Após a leitura dos resumos, resultaram 15 artigos que prosseguiram para leitura na íntegra a fim de avaliar se atendiam a questão de pesquisa. Dois estudos foram excluídos por não corresponderem a temática e um não respondeu a pergunta de pesquisa, sendo assim, incluídos 12 estudos nesta pesquisa, conforme mostra o fluxograma da Figura 1. 
Figura 1 - Diagrama de fluxo PRISMA-ScR evidenciando a busca utilizada para seleção dos resultados. Aracaju, SE, Brasil, 2021.

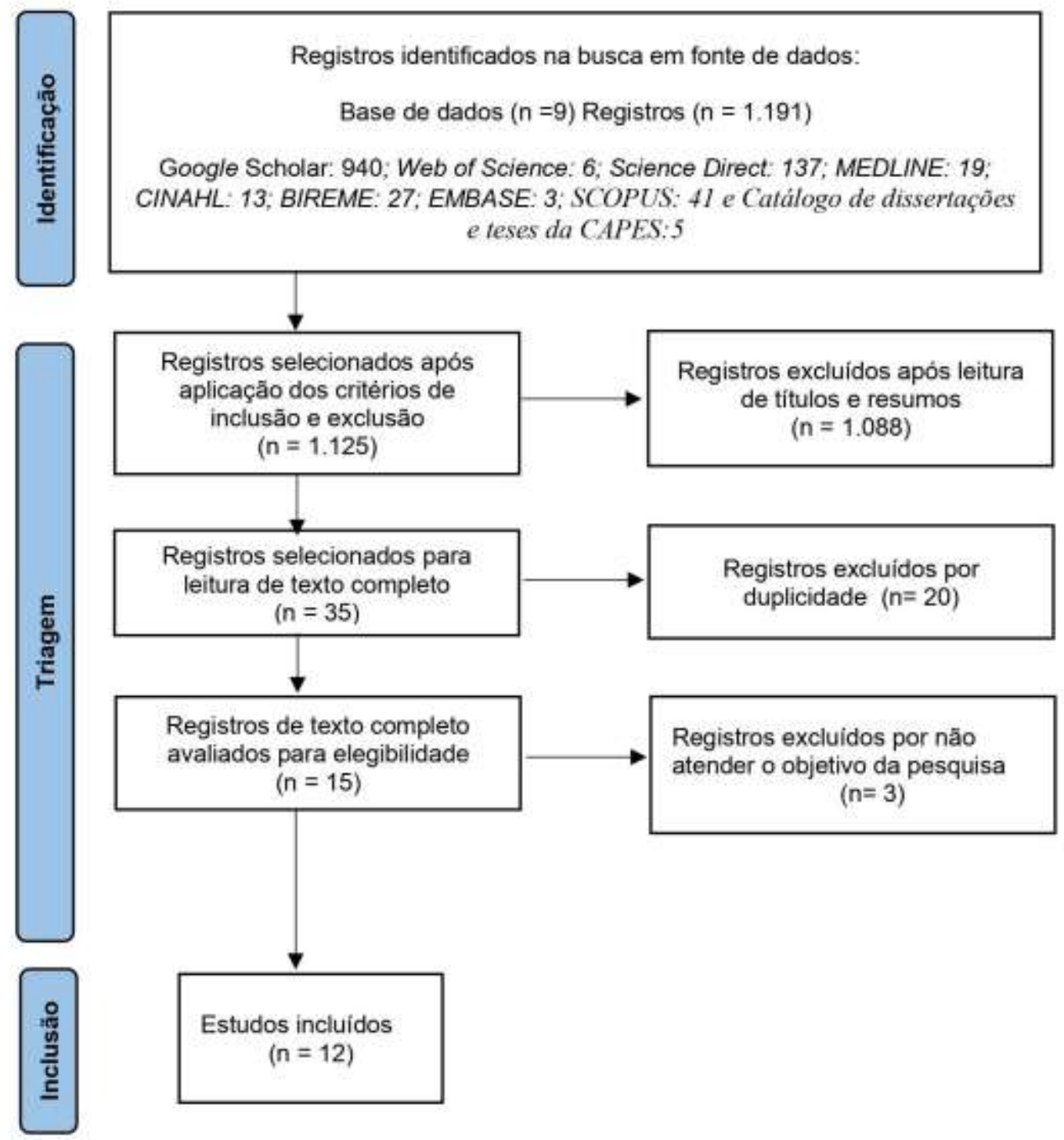

Fonte: Autores (2021).

Entre os 12 registros incluídos na amostra, os lugares que detiveram os maiores números de publicações foram Estados Unidos com sete publicações $(58,3 \%)$ e Brasil com duas (16,6\%) publicações. Em seguida, teve-se México, Egito e Turquia com uma $(8,3 \%)$ publicação cada.

No que se refere ao ano de publicação, 2019, 2016 e 2015 tiveram dois registros publicados em cada ano, após tem-se os anos de 2021, 2018, 2014, 2007, 2006 e 2005 com um artigo incluído. Os doze estudos obtiveram nível de evidência 1B e grau de recomendação A, conforme evidenciado no Quadro 3. 
Quadro 3 - Caracterização das publicações de acordo com título, ano de publicação, país de origem, fonte de dados, nível de evidência e grau de recomendação dos estudos incluídos na scoping review. Aracaju, SE, Brasil, $2021(\mathrm{~N}=12)$.

\begin{tabular}{|c|c|c|c|c|c|}
\hline Autor & Título & $\begin{array}{l}\text { País / } \\
\text { Ano }\end{array}$ & $\begin{array}{l}\text { Fonte de } \\
\text { Dados }\end{array}$ & $\begin{array}{l}\text { Amostra } \\
\text { do } \\
\text { estudo }\end{array}$ & $\begin{array}{l}\text { Nível de } \\
\text { evidência / } \\
\text { Grau de } \\
\text { Recomendação }\end{array}$ \\
\hline Soo et al., 2016 & $\begin{array}{l}\text { Imaging-Guided Core-Needle Breast Biopsy: } \\
\text { Impact of Meditation and Music Interventions on } \\
\text { Patient Anxiety, Pain, and Fatigue. }\end{array}$ & $\begin{array}{l}\text { EUA } \\
2016\end{array}$ & PUBMED & 121 & $\begin{array}{l}\text { Ensaio clínico } \\
\text { randomizado } \\
1 \mathrm{~B} / \mathrm{A}\end{array}$ \\
\hline Domar et al., 2005 & $\begin{array}{l}\text { Relaxation techniques for reducing pain and } \\
\text { anxiety during screening mammography. }\end{array}$ & $\begin{array}{l}\text { EUA } \\
2005\end{array}$ & PUBMED & 143 & $\begin{array}{l}\text { Ensaio Clínico } \\
\text { Randomizado } \\
\text { 1B/A }\end{array}$ \\
\hline Lang et al., 2006 & $\begin{array}{l}\text { Adjunctive self-hypnotic relaxation for outpatient } \\
\text { medical procedures: a prospective randomized } \\
\text { trial with women undergoing large core breast } \\
\text { biopsy. }\end{array}$ & $\begin{array}{l}\text { EUA } \\
2006\end{array}$ & PUBMED & 236 & $\begin{array}{l}\text { Ensaio Clínico } \\
\text { Randomizado } \\
\text { 1B/A }\end{array}$ \\
\hline Wren et al., 2019 & $\begin{array}{l}\text { Preliminary efficacy of a lovinkindness } \\
\text { meditation intervention for patients undergoing } \\
\text { biopsy and breast cancer surgery: A randomized } \\
\text { controlled pilot study }\end{array}$ & $\begin{array}{l}\text { EUA } \\
2019\end{array}$ & CINAHL & 60 & $\begin{array}{l}\text { Ensaio Clínico } \\
\text { Randomizado } \\
\text { 1B/A }\end{array}$ \\
\hline Zavotsky et al., 2014 & $\begin{array}{l}\text { The Effects of Music on Pain and Anxiety During } \\
\text { Screening Mammography }\end{array}$ & $\begin{array}{l}\text { EUA } \\
2014\end{array}$ & CINAHL & 100 & $\begin{array}{l}\text { Ensaio Clínico } \\
\text { Randomizado } \\
\text { 1B/A }\end{array}$ \\
\hline Frank et al., 2007 & $\begin{array}{l}\text { Does therapeutic touch ease the discomfort or } \\
\text { distress of patients undergoing stereotactic core } \\
\text { breast biopsy? A randomized clinical trial }\end{array}$ & $\begin{array}{l}\text { EUA } \\
2007\end{array}$ & SCOPUS & 82 & $\begin{array}{l}\text { Ensaio Clínico } \\
\text { Randomizado } \\
\text { 1B/A }\end{array}$ \\
\hline $\begin{array}{l}\text { Sánchez-Jáuregui, } \\
\text { Téllez, Juárez-García, } \\
\text { García \& García, } \\
2019\end{array}$ & $\begin{array}{l}\text { Clinical Hypnosis and Music In Breast Biopsy:A } \\
\text { Randomized Clinical Trial }\end{array}$ & $\begin{array}{l}\text { MEXIC } \\
\text { O } \\
2018\end{array}$ & $\begin{array}{l}\text { WEB OF } \\
\text { SCIENC }\end{array}$ & 170 & $\begin{array}{l}\text { Ensaio Clínico } \\
\text { Randomizado } \\
\text { 1B/A }\end{array}$ \\
\hline Almeida, 2015 & $\begin{array}{l}\text { Avaliação do impacto de exercícios físicos na } \\
\text { redução da dor em mulheres submetidas à } \\
\text { mamografia }\end{array}$ & $\begin{array}{l}\text { BRASIL } \\
2015\end{array}$ & $\begin{array}{l}\text { CATALOGO } \\
\text { DE } \\
\text { DISSERTAÇ } \\
\tilde{A} O E \text { E TESE } \\
\text { DA CAPES }\end{array}$ & 198 & $\begin{array}{l}\text { Ensaio Clínico } \\
\text { Randomizado } \\
\text { 1B/A }\end{array}$ \\
\hline Leandro, 2016 & $\begin{array}{l}\text { Efeito de um programa de atividade física na } \\
\text { redução da dor durante à mamografia }\end{array}$ & $\begin{array}{l}\text { BRASIL } \\
2016\end{array}$ & $\begin{array}{l}\text { CATALOGO } \\
\text { DE } \\
\text { DISSERTAÇ } \\
\tilde{A} O \text { E TESE } \\
\text { DA CAPES }\end{array}$ & 257 & $\begin{array}{l}\text { Ensaio Clínico } \\
\text { Randomizado } \\
\text { 1B/A }\end{array}$ \\
\hline $\begin{array}{l}\text { Sharaf \& Hafeez, } \\
2019\end{array}$ & $\begin{array}{l}\text { Effect of Nursing Interventions on Pain and } \\
\text { Anxiety among Women undergoing Screening } \\
\text { Mammography }\end{array}$ & $\begin{array}{l}\text { EGYPT } \\
2019\end{array}$ & $\begin{array}{l}\text { GOOGLE } \\
\text { SCHOLAR }\end{array}$ & 100 & $\begin{array}{l}\text { Ensaio Clínico } \\
\text { Randomizado } \\
\text { 1B/A }\end{array}$ \\
\hline $\begin{array}{l}\text { Chelsea Gilts } \\
\text { Ratcliff, } 2015\end{array}$ & $\begin{array}{l}\text { Brief Guided Mindfulness Meditation for Women } \\
\text { Undergoing Stereotactic Breast Biopsy: Effects } \\
\text { on Patient Self-Report, EEG Activity, and } \\
\text { Physiological Measures }\end{array}$ & $\begin{array}{l}\text { EUA } \\
2015\end{array}$ & $\begin{array}{l}\text { GOOGLE } \\
\text { SCHOLAR }\end{array}$ & 76 & $\begin{array}{l}\text { Ensaio Clínico } \\
\text { Randomizado } \\
\text { 1B/A }\end{array}$ \\
\hline
\end{tabular}




\begin{tabular}{|l|l|c|c|c|c|}
\hline & $\begin{array}{l}\text { Effect of music on anxiety and pain during } \\
\text { Akın, 2021 }\end{array}$ & $\begin{array}{c}\text { TURQU } \\
\text { ulrasound-guided core needle breast biopsy: a } \\
\text { randomized controlled trial }\end{array}$ & $\begin{array}{c}\text { IA } \\
\text { SCHOLAR }\end{array}$ & $\begin{array}{c}\text { Ensaio Clínico } \\
\text { Randomizado } \\
1 \mathrm{~B} / \mathrm{A}\end{array}$ \\
\hline
\end{tabular}

Fonte: Autores (2021).

No Quadro 4, encontra-se a sumarização dos resultados dos dados coletados dos registros de acordo com a identificação dos autores, o tipo de método não farmacológico e o desfecho. A música foi testada em cinco estudos, a meditação em três, os exercícios físicos e a hipnose em dois estudos cada e as intervenções de enfermagem e o toque terapêutico em apenas um estudo cada.

Quadro 4 - Sumarização dos resultados de acordo com os autores dos estudos, tipo de método não farmacológico e desfecho.

\begin{tabular}{|c|c|c|}
\hline Autor & $\begin{array}{l}\text { Tipo de método não } \\
\text { farmacológico }\end{array}$ & Desfecho \\
\hline $\begin{array}{c}\text { Soo et al., } 2016 \\
\text { Domar } \text { et al., } 2005 \\
\text { Zavotsky et al., } 2014 \\
\text { Sánchez-Jáuregui et al., } 2019 \\
\text { Akın, } 2021\end{array}$ & Música & $\begin{array}{l}\text { Comparada a meditação guiada, não apresentou redução da dor em } \\
\text { biopsia mamaria guiada por esteriotáxica; } \\
\text { Antes e durante a mamografia não reduz os relatos subjetivos de } \\
\text { ansiedade ou dor; } \\
\text { Reduz apenas a ansiedade e não a dor durante a mamografia; } \\
\text { Pode reduzir a ansiedade, o estresse, a depressão e níveis de dor durante a } \\
\text { biopsia mamária; } \\
\text { Reduziu a ansiedade, mas não a dor durante a biópsia de mama com } \\
\text { agulha grossa guiada por ultrassom; }\end{array}$ \\
\hline $\begin{array}{l}\text { Almeida, } 2015 \\
\text { Leandro, } 2016\end{array}$ & Exercícios físicos & $\begin{array}{l}\text { Exercícios específicos reduzem significativamente a dor durante o exame } \\
\text { mamográfico; } \\
\text { Os exercícios físicos de aquecimento e alongamento em membros } \\
\text { superiores e inferiores pré-exame apresentaram diminuição da percepção } \\
\text { e sensação à dor entre mulheres submetidas a mamografia }\end{array}$ \\
\hline $\begin{array}{c}\text { Soo et al., } 2016 \\
\text { Wren } \text { et al., } 2019 \\
\text { Chelsea Gilts Ratcliff, } 2015\end{array}$ & Meditação & $\begin{array}{l}\text { Mostrou dor significativamente menor durante a biópsia; } \\
\text { Meditação do amor e da bondade melhorou significativamente a dor na } \\
\text { biopsia; } \\
\text { Atenuou o efeito do baixo traço na ansiedade antes e durante a biópsia e } \\
\text { na dor após a biópsia }\end{array}$ \\
\hline $\begin{array}{l}\text { Lang et al., } 2006 \\
\text { Sánchez-Jáuregui et al., } 2019\end{array}$ & Hipnose & $\begin{array}{l}\text { O relaxamento auto-hipnótico reduz a dor e a ansiedade durante a biópsia } \\
\text { de mama com agulha grossa; } \\
\text { Diminuiu significativamente os níveis de dor e depressão em comparação } \\
\text { a música antes da biópsia, mas após não houve diferenças entre os dois } \\
\text { grupos; }\end{array}$ \\
\hline Sharaf \& Hafeez, 2019 & $\begin{array}{l}\text { Intervenções de } \\
\text { enfermagem }\end{array}$ & $\begin{array}{l}\text { Redução estatisticamente significativa nos níveis de dor e ansiedade das } \\
\text { mulheres submetidas à mamografia de rastreamento }\end{array}$ \\
\hline Frank et al., 2007 & Toque terapêutico & $\begin{array}{l}\text { Nenhum benefício significativo do toque terapêutico administrado } \\
\text { durante a biópsia mamária estereotáxica. }\end{array}$ \\
\hline
\end{tabular}

Fonte: Autores (2021).

\section{Discussão}

Este estudo realizou o mapeamento de métodos não farmacológicos utilizados como ferramentas de alívio da dor durante o rastreamento e diagnóstico do câncer de mama, através dos artigos selecionados nas bases de dados que abordassem essa temática. Embora não houvesse limite temporal para esta pesquisa, a inclusão se fez após 2004, o que reforça a atualidade 
do assunto em questão. Além disso, a maior quantidade de publicação de estudos nos Estados Unidos demonstra que este assunto precisa ser mais difundido na comunidade científica.

O método não farmacológico utilizado não foi consenso entre os autores, variou de acordo com o teste nos pacientes, desde musicoterapia, hipnose, meditação, exercícios físicos, toque terapêutico e intervenções de enfermagem. Além disso, é possível perceber que a aplicação desses métodos não se destina apenas ao profissional de enfermagem, mas a médicos radiologistas.

A musicoterapia foi o método mais utilizado nos estudos apresentando divergências de opiniões entre os pesquisadores. Na mamografia, não apresentou efeitos na redução da dor relacionada a compressão mamária na totalidade dos estudos que testaram esse método e reduziu a ansiedade em apenas um dos estudos (Zavotsky et al., 2014, Domar et al., 2005). A realização de exercícios físicos, sejam específicos, de alongamento ou aquecimento pré-exame de mamografia apresenta redução significativa na percepção e sensação dolorosa durante o procedimento (Almeida, 2015, Leandro, 2016). Além disso, as intervenções de enfermagem baseadas na educação em saúde e no ensino de exercícios de respiração profunda apresentaram efeito estatístico significativo na redução da dor e ansiedade, pois 60,0\% das mulheres do grupo de estudo experimentaram dor leve durante o procedimento e 32\% delas relataram sentir-se relaxadas ou não ansiosas (Sharaf \& Hafeez, 2019).

Nos procedimentos de biópsias mamárias, as intervenções testadas encontradas na literatura foram musicoterapia, meditação, hipnose e toque terapêutico. Ressaltamos que efeitos positivos da musicoterapia na redução da dor corresponderam em um estudo apenas (Sánchez-Jáuregui et al., 2019), sendo este resultado contrário ao outro estudo realizado (Akın, 2021). A meditação, testada nos três estudos encontrados, apresentou redução da dor na totalidade dos estudos ressaltando que em um dos estudos, esteve comparada à musicoterapia. Em um dos estudos, o efeito da meditação demonstrou também redução da ansiedade antes e durante o procedimento e, na dor, apenas após a realização da biópsia (Chelsea Gilts Ratcliff, 2015, Soo et al., 2016, Field et al., 2020).

A hipnose quando comparada à música apresentou redução significativa maior da dor antes da biópsia e, após o procedimento, não houve diferença estatística dos métodos, porém ambos eficazes. Além disso, a eficácia do relaxamento auto hipnótico correspondeu também à redução dos níveis de ansiedade apresentados durante a realização de biópsia por agulha grossa (Lang et al., 2006, Sánchez-Jáuregui et al., 2019). Contrariando os efeitos positivos desses métodos utilizados na realização de biópsias mamárias, o toque terapêutico não apresentou nenhum benefício durante a realização de biópsia esteriotáxica (Frank et al., 2007).

Por fim, esses métodos não farmacológicos diversificam o atendimento, possibilitam novas abordagens e experiências, as quais podem ser benéficas ao paciente de maneira a aumentar as taxas de rastreamento e melhorar a experiência das mulheres no diagnóstico do câncer de mama. Além disso, traz conforto ao paciente, diminui ansiedade antes e durante os procedimentos, refletindo no alívio da dor.

É necessário que a partir das informações trazidas nesta revisão de escopo, seja possível aplicar novos estudos com outros métodos não farmacológicos que visem ampliar as estratégias de enfrentamento da dor, confrontar ou reforçar a utilização dos métodos testados nos estudos encontrados, correlacionando o uso das terapias complementares com o cuidado e educação em saúde.

\section{Conclusão}

Esta revisão de escopo permitiu o mapeamento dos métodos não farmacológicos utilizados no alívio da dor durante o rastreamento e diagnóstico do câncer de mama. Através das evidências trazidas neste estudo, foi possível ver que não há uma variedade de estratégias utilizadas, no entanto, os estudos mostraram maiores resultados no alívio da dor nas biópsias mamárias. A meditação, a hipnose e a música apresentaram efeito no alívio da dor durante a realização das biópsias mamárias 
e, na mamografia, apenas a realização de exercícios físicos e as intervenções de enfermagem mostraram efeito significativo. Diante do baixo número de métodos não farmacológicos testados e pequenos resultados apresentados durante a mamografia, sugere-se novos estudos com outras estratégias, uma vez que a dor da compressão mamária é um limitador à adesão.

Espera-se que o mapeamento desses métodos possa contribuir para mudanças nos protocolos de atendimentos desses procedimentos por ser uma ferramenta de fácil acesso, baixo custo, criativa, segura, que fornece benefícios às mulheres. Sugere-se realização de novos estudos com abordagem metodológica e grupos populacionais distintos que possam contribuir com mais evidências científicas

\section{Referências}

Abdel-Hadi, M., Abdel-Hamid, G. F., Abdel-Razek, N., \& Fawzy, R. K. (2010). Should fine-needle aspiration cytology be the first choice diagnostic modality for assessment of all nonpalpable breast lesions? The experience of a breast cancer screening center in Alexandria, Egypt. Diagnostic Cytopathology, 38(12), $880-889$.

Akın, M. E. (2021). Effect of music on anxiety and pain during ultrasound-guided core needle breast biopsy: a randomized controlled trial. Diagn Interv Radiol, 27, 360-365.

Almeida, T. C. (2015). Avaliação do impacto de exercícios físicos na redução da dor em mulheres submetidas à mamografia. Tese de Doutorado, Faculdade de Medicina de Ribeirão Preto, Ribeirão Preto, SP, Brasil.

Almeida, L. S., Santana, J. B., Silva, S. O., \& Melo, M. I. B. (2017). Acesso Ao Exame De Mamografia Na Atenção Primária. Rev enferm UFPE on line, 11, 4885-94.

Domar, A. D., Eyvazzadeh, A., Allen, S., Roman, K., Wolf, R., Orav, J., Albright, N., \& Baum, J. (2005, Fev). Relaxation techniques for reducing pain and anxiety during screening mammography AJR Am J Roentgenol, 184(2), 445-7.

Chelsea Gilts Ratcliff, M.A. (2015). Brief Guided Mindfulness Meditation for Women Undergoing Stereotactic Breast Biopsy: Effects on Patient Self-Report, EEG Activity, and Physiological Measures. Tese de Doutorado, University of Houston, Texas, Estados Unidos.

Fayanju, O. M., Kraenzle, S., Drake, B. F., Oka, M., \& Goodman, M. S. (2014). Perceived barriers to mammography among underserved women in a Breast Health Center Outreach Program. American Journal of Surgery, 208, n425-434.

Field, A. S., Raymond, W. A., Rickard, M., \& Schmitt, F. (2020, March-April). Breast fine needle aspiration biopsy cytology: the potential impact of the International Academy of Cytology Yokohama System for Reporting Breast Fine Needle Aspiration Biopsy Cytopathology and the use of rapid on-site evaluation. Journal of the American Society of Cytopathology, 9, 103-111.

Frank, L. S., Frank, J. L., Marc, D., Makari-Judson, G., Barham,, R. B., \& Mertens, W, C. (2007). Does therapeutic touch ease the discomfort or distress of patients undergoing stereotactic core breast biopsy? A randomized clinical trial. Pain Med, 8 (5), 419-24.

Freitas-junior, R., Martins, E., Metran-Nascente, C., Carvalho, A. A., Silva, M. F., Soares, L. R., \& Ximenes, C. A. (2018). Double-blind placebo-controlled randomized clinical trial on the use of paracetamol for performing mammography. Medicine (United States), 97 (13).

Gioia, S., Lima, S., \& Maiato, A. P.A. (2017). Biópsia percutânea de mama: quando indicar?. Sociedade Brasileira de Mastologia.

Hamlin, A. S.; \& Robertson, T. M. (2017). Pain and Complementary Therapies. Critical Care Nursing Clinics of North America, $29,449-460$.

Instituto Nacional do Câncer José Alencar Gomes da Silva Atualização em mamografia para técnicos em radiologia / Instituto Nacional do Câncer José Alencar Gomes da Silva. (2a ed.), INCA.

Kitaoka, H.; \& Kawashima, H. (2018). Influence of the menstrual cycle on compression-induced pain during mammography: correlation with the thickness and volume of the mammary gland. Radiological Physics and Technology, 11, 20-26.

Lang, E. V., Berbaum, K, S., Faintuch, S., Hatsiopoulou, O., Halsey, N., Li, X., Berbaum, M. L., Laser, E., \& Baum, J. (2006). Adjunctive self-hypnotic relaxation for outpatient medical procedures: a prospective randomized trial with women undergoing large core breast biopsy. Pain, $126(1-3), 155-64$.

Le danseur, M., Crow, A. D., Stutzman, S. E., Villarreal, M. D., \& Olson, D. M. (2019). Music as a Therapy to Alleviate Anxiety during Inpatient Rehabilitation for Stroke. Rehabilitation Nursing, 44, 29-34.

Leandro, R. I. S. (2016). Efeito de um programa de atividade física na redução da dor durante à mamografia. Dissertação, Faculdade de Medicina, Universidade Federal do Ceará, Fortaleza, CE, Brasil.

Oxford Centre for Evidence-Based Medicine [Internet]. (2009, March). Levels of Evidence https://www.cebm.net/2009/06/oxford-centre-evidence-basedmedicine-levels-evidence-march-2009/.

Pang, E., Crystal, P., Kulkarni, S., Murphy, K., \& Menezes, R. J. (2016, Agosto). An Audit of Pain Experienced During Image-Guided Breast Biopsy Procedures at an Academic Center. Jornal da Associação Canadense de Radiologistas, Volume 67, edição 3, páginas 250-253.

Peters, M.D.J., Godfrey, C., McInerney, P., Munn, Z., Tricco, A.C., \& Khalil, H. (2020). Chapter 11: Scoping Reviews (2020 version). In: Aromataris E, Munn Z (Editors). Joanna Briggs Institute Reviewer’s Manual, JBI. https://reviewersmanual.joannabriggs.org/. 
Research, Society and Development, v. 10, n. 10, e446101019173, 2021

(CC BY 4.0) | ISSN 2525-3409 | DOI: http://dx.doi.org/10.33448/rsd-v10i10.19173

Satchithananda, K., Fernando, R. A., Ralleigh, G., Evans, D. R., Wasan, R. K., Bose, S., Donaldson, N., \& Michell, M. J. (2005, Nov-Dez). Uma auditoria da dor / desconforto experimentado durante procedimentos de biópsia de mama guiada por imagem. Mama J., 11 (6): $398-402$.

Sánchez-Jáuregui, T., Téllez, A., Juárez-García, D., García, C. H., \& García, F. E. (2019). Clinical Hypnosis and Music In Breast Biopsy:A Randomized Clinical Trial. Am J Clin Hypn, 61(3), 244-257.

Sharaf, A. Y., \& Hafeez, N. A. A. A. E. (2019, September - December). Effect of Nursing Interventions on Pain and Anxiety among Women undergoing Screening Mammography. International Journal of Novel Research in Healthcare and Nursing, 6, 454-469.

Soo, A. E., Shelby, R. A., Miller, L. S., Balmadrid, M. H., Johnson, K. S., Wren, A. A., Yoon, S. C., Keefe, F. J., \& Soo, M. S. (2014, Jul). Predictors of pain experienced by women during percutaneous imaging-guided breast biopsies. J Am Coll Radiol, 11(7):709-16.

Soo, M. S., Jarosz, J. A., Wren, A. A., Soo, A. E., Mowery, Y. M., Johnson, K. S., \& Shelby, R. A. (2016). Imaging-Guided Core-Needle Breast Biopsy: Impact of Meditation and Music Interventions on Patient Anxiety, Pain, and Fatigue. Journal of the American College of Radiology, 13(5), 526-534.

Tricco, AC., Lillie E., Zarin, W., O’Brien, K.K., Colquhoun, H., Levac, D., Moher, D., Peters, M. D. J., Horsley, T., Weeks, L., Hempel, S., Akl, E. A., Chang, C., McGowan, J., Stewart, L., Hartling, L., Aldcroft, A., Wilson M. G., Garritty, C., Lewin, S., Godfrey C. M., Macdonald, M. T., Langlois, E. V., Soares-Weiser, K., Moriarty, J., Clifford, T., Tunçalp, O., \& Straus, S. E. (2018). PRISMA Extension for Scoping Reviews (PRISMA-ScR): checklist and explanation. Ann Intern Med.; 169(7):467-73.

Wren, A. A., Shelby, R. A., Soo, M. S., Huysmans, Z., Jarosz, J. A., \& Keefe, F. J. (2019). Preliminary efficacy of a lovingkindness meditation intervention for patients undergoing biopsy and breast cancer surgery: A randomized controlled pilot study. Support Care Cancer, 27(9), 3583-3592.

Yangfan, X., Li, L., Xie, Y., Xu, J., \& Liu, Y. (2018). Effects of aroma therapy and music intervention on pain and anxious for breast cancer patients in the perioperative period. Journal of Central South University (Medical Sciences), 43, 656-661.

Zagouri, F., Sergentanis, T. N., Gounaris, A., Koulocheri, D., Nonni, A., Domeyer, P., \& Zografos, G. C. (2008). Dor em diferentes métodos de biópsia mamária: Ênfase na biópsia mamária assistida a vácuo. The Breast, 17 (1), 71-75.

Zavotsky, K. E., Banavage, A., James, P., Easter, K., Pontieri-Lewis, V., \& Lutwin, L. (2014, Jun). The effects of music on pain and anxiety during screening mammography. Clin J Oncol Nurs, 18(3), E45-9. 